\title{
Mass Spectrometry for the study of natural mechanisms
}

T he 16th French Mass Spectrometry Meeting, held in Nancy in 1999, has covered various scientific areas such as environment, biology, wine science, medicine, geology, chemistry, archaeology and reaction mechanisms. Selected papers from this meeting are published in this issue of EurJAC and also in special issues of the journals Organic Geochemistry and European Mass Spectrometry. In this dossier, most investigations are based on the recently developed ElectroSpray Ionisation method (ESI-MS), which is a very promising tool because it allows the identification of water-soluble, thermally sensible, high-molecular compounds, whereas older methods such as GC-MS are restricted to low-molecular weight, volatile substances. Moreover, ESI-MS is particularly relevant to biological studies involving host-guest recognition because complexes formed by weak, noncovalent binding are preserved into the mass analyser. A neat example of such interactions is presented by Nierengarten et al. who tagged enantiomeric hosts in order to study their complexation with crown ethers.

ESI-MS has also rapidly found applications in the growing field of combinatorial chemistry. Here, this technique is particularly well suited to identify the structure of soluble polymers used to perform chemical reactions, as underlined by Varray et al. who studied polyethyleneglycol oligomers of average mass of 2000-3400 and their derivatives. Vivet et al further show that the soft ionisation conditions of ESI-MS can be optimised to analyse thermally sensible peptides of therapeutic interest built by condensation of up to 13 amino-acids. Another elegant biomedical application of ESI-MS is presented by Rathahao et al. who studied mutagenic catechols and glutathione conjugates formed during the metabolism of the estradiol hormone. Rochut et al. further demonstrate that ESI-MS can be used to distinguish natural cattle proteins (somatotropins) from recombinant analogues used to artificially increase milk production of dairy cows. Enoui et al. identify by ESI-MS aldehydic metabolites which may be involved in human diseases such as cancer. In an investigation of mutagenic polycyclic aromatic hydrocarbons (PAHs) produced by vehicle exhausts, Bryselbout et al. show by GC-MS the existence of an atmospheric distillation effect occurring near highways.

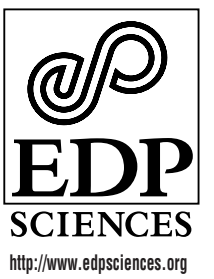

Coordinated by: É. Lichtfouse Institut Soil and Environment Laboratories, INRA-ENSAIA/NPL, BP 172, 